\title{
¿Cómo fallan y controlan la ejecución de las penas sustitutivas los jueces?
}

\author{
Ana María Morales Peillard* \\ Sebastián Salinero Echeverría**
}

\begin{abstract}
RESUMEN
El presente estudio indaga mediante entrevistas semiestructuradas practicadas a una muestra de jueces de garantía y oral, acerca de las concepciones penológicas de los jueces y busca establecer en qué medida estas influirían la decisión de juzgamiento entre una pena de cárcel y una sustitutiva. Además, explora las percepciones acerca de la labor de control de la ejecución de las penas. Los hallazgos dan cuenta de un proceso complejo de juzgamiento en donde las percepciones penológicas tendrían una influencia menor y en el que se estaría mermando el deber de motivación de las sentencias. En materia de ejecución penal, por su parte, el estudio revela la importancia de contar con herramientas para su control, y la necesidad de contar con una judicatura abocada exclusivamente al conocimiento de dicha temática.
\end{abstract}

Alternativas a la cárcel; juzgamiento; percepción judicial

$$
\begin{gathered}
\text { ¿How do judges sentence and control de execution } \\
\text { of alternatives sanctions? }
\end{gathered}
$$

\begin{abstract}
The present study inquiries through semistructured interviews with a sample of guarantee and oral judges, about the penological conceptions of the judges and to establish to what extent these would influence the sentencing decision between a prison sentence and an alternative sanction. In addition, it explores their perceptions about the work of controlling the execution of sentences.
\end{abstract}

* Licenciada en Ciencias Jurídicas y Sociales, Universidad de Chile. Abogada. Msc in Criminal Justice Policy, London School of Economics and Political Science, Inglaterra. Directora Área de Justicia y Reinserción de la Fundación Paz Ciudadana. Docente en Criminología y Política Criminal, Facultad de Derecho, Universidad Alberto Hurtado. Correo electrónico: amorales@pazciudadana.cl

** Abogado. Magíster en Derecho Penal, Universidad de Talca, Chile. Doctor en Derecho, Universidad de Lérida, España. Profesor de Derecho Penal y Criminología, Facultad de Derecho, Universidad de Talca, Chile.Correo electrónico: ssalinero@utalca.cl

El texto es parte del proyecto Fondecyt No 1160970 , titulado "Evaluación de las penas sustitutivas y el camino hacia una política criminal moderna de alternativas a la cárcel”. Agradecemos los comentarios efectuados por el magistrado Fernando Guzmán a borradores del presente artículo.

Artículo recibido el 25.7.2019 y aceptado para su publicación el 27.2.2020. 
The findings gave an account of a complex sentencing process in which penological perceptions would have a lesser influence and in which the duty to motivate sentences would have been depleted. In the execution of the sentence arena, the study gives reveals the importance of having tools to control it and the need to have a judiciary dedicated exclusively to the knowledge of this topic.

Alternatives to prison; sentencing; judicial perception

\section{INTRODUCCIÓN}

En Chile, la Ley 20.603, publicada el 27 de junio de 2012, introdujo importantes modificaciones a la Ley 18.216 acerca de medidas alternativas a la privación o restricción de libertad. Esta reforma legal importó, entre otras cosas, una nueva nomenclatura para los otrora "beneficios", pasando a tener la modalidad de castigo sustitutivo de las penas privativas o restrictivas de la libertad y ampliándose el catálogo de alternativas susceptibles de ser impuestas.

Han transcurrido más de 5 años desde el inicio de la puesta en marcha de dicha reforma ${ }^{1}$, sin embargo hasta la fecha no se cuenta con investigaciones empíricas acerca de su funcionamiento judicial y cómo es percibida por los actores. En ese contexto, la presente investigación buscó conocer la percepción penológica de los jueces y comprender en qué medida la labor de juzgamiento y de fundamentación de las penas sustitutivas se vería influía por dichas construcciones. Junto con lo anterior, la investigación indagó acerca de sus percepciones en torno a la labor de control de la ejecución, considerando que se trata de una de las innovaciones contenidas en la ley.

\section{Acerca de labor de juzgamiento}

\section{Aproximaciones teóricas}

Lovegrove postuló la posibilidad de establecer ciertas "leyes de juzgamiento" que permitieran predecir el resultado de los fallos judiciales, basados en la existencia de una cierta causalidad entre factores que tuvieran un elevado valor predictivo, particularmente la gravedad del delito y la existencia de condenas anteriores ${ }^{2}$. Probablemente, un proceso así de nítido y predecible sea la ambición de políticos y legisladores, buscando de esa forma restringir la desdeñada discreción judicial. Sin embargo, tal afirmación es problemática en la medida que se basa en el supuesto que la sentencia es racional y que, si se analiza lo suficiente, es posible descubrir un modelo de sentencia. Como indica Hutton, lo anterior confunde la existencia de patrones con la racionalidad, mientras que el hecho de que

${ }^{1}$ La ley postergó su entrada en vigencia a la fecha de dictación de su reglamento contenido en el Decreto No 1120 del Ministerio de Justicia, de 23 de diciembre de 2013.

${ }^{2}$ Lovegrove, 1989, p. 47 y ss. 
exista un patrón no implica que exista necesariamente un modelo racional de sentencia que sea operado inconscientemente por los juzgadores ${ }^{3}$. En efecto, dicho ejercicio omite el hecho de que los jueces son actores sociales, que se perciben reflexivamente a sí mismos como personas que toman decisiones, aunque dentro de ciertos límites ${ }^{4}$.

Tomando como base dichos planteamientos, diversas investigaciones que han buscado entender el proceso de juzgamiento, han encontrado que las sentencias que imponen los jueces, así como las potenciales disparidades entre las mismas, están fuertemente relacionadas con la filosofía penal, es decir, las preferencias penológicas ${ }^{5}$ y las actitudes de los jueces ${ }^{6}$. Dicha filosofía penal se enmarca en lo que Ashworth denomina "influencias informales" del proceso de juzgamiento ${ }^{7}$ que se distinguirían de las "fuentes formales" 8 . Así, las últimas estarían típicamente compuestas -en el sistema de common law descrito por el autor- por la legislación, las "guías de sentencias" y las decisiones judiciales. Asimismo, las "influencias informales" de cierta forma vendrían a permear la decisión de juzgamiento por medio de factores extralegales que representan la visión del juzgador derivada de su experiencia en tal rol, sus propias construcciones y creencias acerca de la dogmática penal y de la realidad criminal, unida a la existencia de ciertas características sociodemográficas?

Este tipo de distinciones dan cuenta que la labor de juzgamiento y sus resultados no son solo producto de la aplicación de reglas legales ni pueden explicarse únicamente por factores como la gravedad del delito cometido o la historia criminal, sino que son producto de filosofías preconcebidas y actitudes que de cierta forma predisponen a los jueces encargados de la labor de juzgamiento y los hacen responder a los distintos casos a los que se ven enfrentados basados en sus creencias personales acerca de los fines de cada pena y sus adecuaciones ${ }^{10}$.

\footnotetext{
${ }^{3}$ Hutton, 2006, p. 159.
}

${ }^{4}$ Hutton, 2006, p. 159.

${ }^{5}$ Las preferencias penológicas refieren a la adscripción a las teorías tradicionales acerca del castigo: las absolutas o de retribución y las teorías relativas, prevencionistas, o utilitaristas; pudiendo distinguirse entre estas últimas aquellas relativas a la prevención general o disuasión y aquellas referidas a la prevención especial, ya sea en su dimensión positiva o de reinserción social, o en la negativa o de incapacitación. Para un análisis profundo de estas teorías y las visiones contemporáneas de naturaleza híbrida véase, entre otros, Frase, 2005, p. 65 y ss.; Roxin, 2006; Feijoo, 2007 y Lippke, 2006, p. 273-295.

${ }^{6}$ Hogarth, 1971, p. 68 y ss; Mackenzie, 2005, p. 89 y ss; De Castro Rodríguez et al., 2019, pp. 174-175.

${ }^{7}$ Ashworth, 2010, p. 41.

8 AshwORTH, 2010, p. 24.

9 Ashworth, 2010, p. 43-44.

${ }^{10}$ Hogarth, 1971, p. 68 y ss; Mackenzie, 2005, p. 89 y ss; De Castro Rodríguez et al., 2019, pp. 174-175. 


\section{Aproximaciones empíricas}

La literatura criminológica da cuenta de investigaciones tanto de tipo cuantitativas como cualitativas que han buscado explorar cómo operan las influencias informales en el proceso de juzgamiento, centrándose su análisis en factores extralegales particulares buscando determinar su influjo. De acuerdo con Ulmer, estos estudios incluyen, mas no se encuentran limitados, a las siguientes temáticas: a) estratificación social e inequidad en el juzgamiento; b) ejercicio de la discreción judicial y el proceso de toma de decisiones por parte de los jueces; c) la realidad práctica del juzgamiento y su contraste con los objetivos político-criminales y la regulación; y d) la influencia y adaptación a las realidades locales y contextos sociales ${ }^{11}$.

En el caso del primer grupo de investigaciones, estas se han centrado en estudiar en qué medida las decisiones de juzgamiento vienen moldeadas y de cierta manera reproducen patrones de estratificación social e inequidad, basadas en la influencia de la raza, etnicidad, género u otros factores similares. Estos estudios generalmente han utilizado viñetas o ejercicio de juzgamiento ficticios y la recolección de datos estadísticos de distintas bases que permitan mediante técnicas cuantitativas aislar el efecto de un determinado factor, controlando el efecto del resto ${ }^{12}$. La mayoría de esos estudios han encontrado enormes disparidades basadas en la raza u origen social ${ }^{13}$.

El segundo grupo de investigaciones, que se encuentra íntimamente vinculado con el segundo y el cuarto grupo mencionado por Ulmer ${ }^{14}$, ha buscado dar cuenta de las complejidades del proceso de juzgamiento mediante estudios mixtos o puramente cualitativos, los que utilizando técnicas etnográficas ${ }^{15}$ han buscado explorar el proceso entendiendo al mismo como una "práctica social" ${ }^{16}$. Si bien el acento en los diferentes factores extralegales difieren entre las distintas investigaciones, en términos generales concuerdan en la importancia de estas variables contextuales para explicar la discreción judicial y el proceso de toma de decisiones para el juzgamiento; sin embargo, su hallazgo más importante ha sido el de develar la influencia de la "cultura judicial" (bench culture) ${ }^{17}$, dando cuenta que la naturaleza social de la función judicial genera la existencia de una cultura distintiva ${ }^{18}$.

Por último, encontramos la tercera categoría de estudios descrito por Ulmer -dentro de las que se enmarca la presente investigación-, que han buscado indagar acerca de la percepción de los jueces respecto de los principios fundantes de la teoría penal, y sus

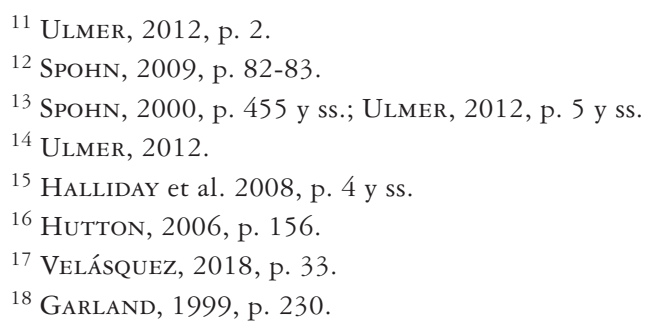


visiones pertinentes al delito y el castigo y su función al imponer la sentencia, buscando además indagar eventuales distancias o brechas entre la regulación y la práctica ${ }^{19}$.

Una de las primeras investigaciones en esta temática fue la realizada por Hogarth en 1971, el que mediante diversas técnicas tanto cualitativas (entrevistas) como cuantitativas (encuestas, uso de instrumentos de autorreporte y análisis de información secundaria) buscó "mirar el juzgamiento a través de los ojos de los magistrados" ${ }^{20}$, teniendo como objetivo central buscar y conocer las preferencias de doctrina penal de los jueces. Los hallazgos de las entrevistas mostraron que la doctrina más popular declarada por los magistrados era la rehabilitación, seguida de la disuasión general, la disuasión individual y la incapacitación, ubicando la retribución en último lugar. En términos de las sanciones, la probation y el confinamiento en lugares especiales eran percibidas como aquellas efectivas para lograr la reforma de los infractores al igual que el mecanismo de la libertad condicional. Asimismo, en términos de la coherencia, si bien la mayoría de los jueces tenía la imagen de sí mismos como orientado a los fines de rehabilitación, en la práctica sus tomas de decisión estaban más bien fundadas en el delito cometido y la protección de la comunidad ${ }^{21}$.

Otro estudio relevante fue el realizado por Mackenzie, el que, a base de entrevistas a jueces practicadas en Australia, examinó sus preferencias penológicas, encontrando un prácticamente nulo soporte al fin de retribución por parte de los jueces, los que privilegiaban fines de rehabilitación, buscando atender al impacto de la sentencia en los infractores y en la sociedad en general. Sin embargo, los hallazgos dieron cuenta que no obstante privilegiar dichos fines, estos eran generalmente considerados a la hora de imponer penas alternativas, mas no tratándose de penas de cárcel. Sin embargo, la autora encontró que en muchos casos en los que los jueces declararon fallar basados en consideraciones disuasivas generales, lo cierto es que en la práctica su fundamentación se acercaba más a consideraciones retributivas ${ }^{22}$.

Importante fue también el estudio desarrollado por Hough et al. en Inglaterra, el que mediante una investigación predominantemente cualitativa documentó el proceso de juzgamiento de los jueces en los casos límites (borderline cases), es decir, aquellos ponen en la posición al juzgador de tener que decidir entre la aplicación de una pena de prisión y sanciones alternativas ${ }^{23}$. Los hallazgos mostraron que los jueces, por lo general, prefieren enviar a los condenados a la cárcel solo como "último recurso", cuando la gravedad de los delitos o los antecedentes penales de los infractores no les dejara ninguna opción ${ }^{24}$. Lo anterior implica que las sanciones alternativas presentan mejores opciones de sentencia para la mayoría de los casos, de acuerdo con lo reportado por los jueces. Estos hallazgos pueden vincularse a los resultados de investigaciones más recientes como la realizada

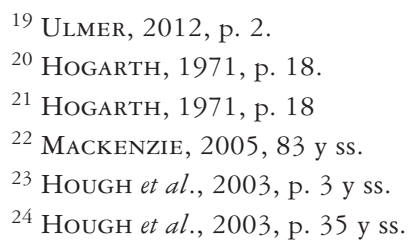


por Maguire ${ }^{25}$ y Tata ${ }^{26}$, quienes argumentan que el concepto de "último recurso" es fácilmente manipulable por los jueces y que en definitiva hace que la cárcel sea la única sanción que en realidad no requiere ser fundamentada mediante un fin específico, pues siempre puede ser impuesta bajo el argumento de que ninguna otra justificación resulta plausible para el caso.

\section{El JUZGamiento y CONTROL DE LA EJECUCión DE LAS ALTERnativas a LA CÁRCEL EN CHILE}

La labor de juzgamiento de las penas sustitutivas se encuentra encomendada, desde la implementación de la reforma procesal en 2000, a los jueces penales, pudiendo ser realizada por jueces de garantía (en adelante JG), tratándose de los delitos menores o de mediana gravedad por medio del procedimiento monitorio, simplificado o abreviado; mientras que los delitos más graves deben ser juzgados por los jueces orales (en adelante JO) pertenecientes a los Tribunales Orales en lo Penal (en adelante TOP), los que actúan colegiadamente ${ }^{27}$. Por su parte, la labor de control de la ejecución o cumplimiento de las penas se encuentra encomendada exclusivamente a los JG, de tal forma que si bien un caso puede ser juzgado por un TOP, el control del cumplimiento regresa al tribunal donde se controló la investigación, aun cuando excepcionalmente esta labor puede ser ejercida por el JG del lugar donde deba cumplirse la pena ${ }^{28}$.

Para la labor de juzgamiento, el juez debe ceñirse a las disposiciones del Código Procesal Penal (en adelante, CPP), Código Penal (en adelante, CP), estableciendo este último como penas principales la pena privativa de la libertad y la multa. No obstante, también se contempla la posibilidad de sustituir la pena carcelaria, en los casos en que la pena a imponer no sea superior a 5 años y se dé cumplimiento a ciertos requisitos. Dicha posibilidad se encuentra regulada en la Ley 18.216, antes denominada de "medidas alternativas a las penas privativas de la libertad", la que sufrió una reforma en 2012 en virtud de la Ley 20.063, pasando a renombrarse como "Penas sustitutivas" y reconociéndose como penas las siguientes: remisión condicional, reclusión parcial, libertad vigilada, libertad vigilada intensiva, la expulsión de extranjeros sin residencia legal y la prestación de servicios en beneficio de la comunidad (en adelante, PSBC).

En términos de la justificación penológica, si bien nuestro CP es marcadamente retribucionista, debido a que el castigo está establecido en una escala en función de la gravedad de la ofensa, reservándose la cárcel justamente para aquellas que están en la escala superior -más graves-, pudiendo solo aquellas de baja y mediana gravedad ser acreedores del sistema de sanciones alternativos a la prisión ${ }^{29}$; los lineamientos penológicos de esta

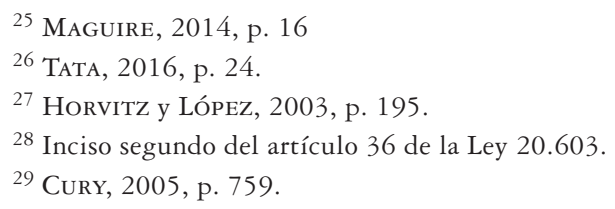


reforma apuntan a concebir las penas sustitutivas bajo prismas preventivos especiales positivos. Así, el mensaje del proyecto de ley que dio inicio a su tramitación efectuó una apuesta clara por la reinserción social, estableciendo como objetivo de estas penas el de evitar la reincidencia delictual ${ }^{30}$. En ese sentido, si bien la inspiración penológica de la reforma sigue la línea ya trazada por la Ley 18.216 originaria -también de corte preventivo especial positiva-, difiere en cuanto a la concepción acerca de la reinserción social que adopta. Así, la conceptualización primigenia de las penas alternativas parece más responder a una "lectura mínima y garantista" 31 más que imponer un contenido positivo y concreto a los conceptos de reinserción social, o sea, busca evitar el efecto disocializador de las penas privativas de la libertad, siendo esencial la evitación de los aspectos más nocivos de la estancia en la cárcel ${ }^{32}$; mientras que la reforma no solo recogería dicha lectura minimalista, sino que de la historia de la ley se desprende una concepción más cercana a la lógica del What works (Qué funciona), que asume que la reinserción social se alcanza abordando las causas que llevaron al infractor a delinquir, buscando reducir la criminalidad futura del infractor mediante el tratamiento en la comunidad ${ }^{33}$.

Por otra parte, para efectos de determinar su procedencia, pesa sobre el sentenciador el deber de fundamentación, que en el ámbito de las penas sustitutivas supone un pronunciamiento acerca de la concurrencia o no de los requisititos establecidos en la ley. Particularmente, del análisis normativo de las penas sustitutivas se pueden advertir la existencia de requisitos de tipo objetivos y subjetivos.

Tratándose de los requisitos objetivos, para todas las penas sustitutivas el criterio a identificar dice relación con el quantum de la pena privativa o restrictiva de libertad, que difiere respecto de cada pena y el tipo de delito por el que se condena, considerando que hay delitos absoluta o relativamente excluidos de la aplicación de las penas sustitutivas ${ }^{34}$.

Los requisitos subjetivos son aquellos que miran ciertas características del sujeto condenado, y se subclasifican en aquellos que denominaremos de "peligrosidad objetiva" (de constatación) y que se ocupan fundamentalmente de regular las hipótesis en que se admite o no reincidencia previa; y aquellos de peligrosidad subjetiva (de prognosis), en donde el juez debe fundamentar que posiblemente el condenado no volverá a delinquir, y que el tratamiento - extramuros- o bien la ausencia de tratamiento será idóneo o suficiente para alcanzar la reinserción social. Es importante señalar que estos supuestos no son homónimos en las diversas penas sustitutivas, sino que ellos varían entre ellas ${ }^{35}$.

\footnotetext{
30 Véase Biblioteca del Congreso, 2012, pp. 5-6 y 20.

31 Mapelli, 1983. Cit: Medina, 2011, pp. 154 y ss.

32 Morales y Salinero, 2019, p. 9.

33 Para una discusión más acabada acerca de los fines político-criminales de las alternativas a la cárcel en Chile a lo largo de su historia, véase Morales y Salinero, 2019, p. 1 y ss.

34 Artículo 1 Ley $\mathrm{N}^{\circ} 20.603$.

${ }^{35}$ Véase letras c) y d) del artículo 4, letra c) del artículo 8; letra b) del artículo 10 e inciso final del artículo 15 de la Ley $\mathrm{N}^{\circ} 20.603$.
} 
Junto con lo anterior, para efectos de la labor de ejecución desde la perspectiva jurisdiccional, la Ley 20.603 le entrega herramientas novedosas al juez: la realización de audiencias de seguimiento, la regulación de un procedimiento progresivo de respuesta en caso de incumplimiento, y la posibilidad de reemplazar la pena sustitutiva por una menos gravosa en caso de cumplimiento de más de la mitad de la pena.

En el primer caso, con ocasión de la regulación de la libertad vigilada simple e intensiva, la ley contempla en el inciso tercero del artículo 23 el deber del tribunal de convocar a audiencias de seguimiento, de carácter anual en el caso de la primera y de carácter semestral en el caso de la segunda; audiencias que junto con los informes semestrales y trimestrales que -respectivamente- deben remitir al tribunal los delegados, buscan que el juez cuente con información acerca del cumplimiento de la sanción mientras esta es ejecutada, y no solo en caso de incumplimiento o revocación. Asimismo, en el caso de la PSBC, la ley establece en el artículo 13 bis, la facultad del juez -de oficio o a solicitud del condenado- de efectuar un control respecto de las condiciones de su cumplimiento, debiendo citar, en ese caso, a una audiencia de seguimiento durante el período que dure su ejecución. La diferencia entre las audiencias de seguimiento tratándose de la libertad vigilada simple o intensiva y la de la PSBC, es que en este último caso su realización es facultativa, mientras que tratándose de la libertad vigilada en cualquiera de sus modalidades, su realización se encuentra compelida por ley.

Si bien las referidas audiencias de seguimiento contempladas en la ley no cuentan con un homólogo en nuestra legislación, estas ya encontraban albergue en la praxis judicial, con ocasión de las audiencias realizadas en los tribunales participantes de programa de Tribunales de Tratamiento de Drogas (en adelante, TTD), impulsado en el marco de un convenio interinstitucional suscrito por diversas instituciones ${ }^{36}$.

Por su parte, la ley contempla en el artículo 24 y siguientes un régimen de intensificación en caso de incumplimiento de determinadas condiciones, otorgando facultades al tribunal para intensificar la pena originalmente impuesta, revocarla por otra pena sustitutiva más gravosa o en último término revocarla por la cárcel, en casos graves o reiterados de las condiciones impuestas. Como contrapartida, el artículo 32 establece para los casos en que hubiere transcurrido más de la mitad de la condena, la posibilidad -previo informe de Gendarmería de Chile- de sustituirla por una pena menos gravosa. Al respecto, resulta pertinente señalar que las herramientas de intensificación y reemplazo de la pena originalmente impuestas son novedosas en la medida que no se encontraban contempladas en la antigua regulación de las "medidas alternativas", aun cuando reglas de similar tenor ya se encontraban introducidas en la Ley $20.084^{37}$.

Respecto de su funcionamiento empírico, vale la pena mencionar que en la práctica, solo los casos graves son juzgados ante los TOP, mientras que más del $90 \%$ de los casos son

36 Véase convenio interinstitucional acerca de Tribunales de Tratamiento de Drogas, suscrito el 29 de marzo de 2012.

${ }^{37}$ Véase los artículos 52 y 53 de la Ley 20.084. 
conocidos y juzgados por los $\mathrm{JG}^{38}$ mediante procedimientos simplificados o abreviados, por lo que es razonable presumir que la mayoría de las sentencias a penas sustitutivas son dictadas por estos últimos. Lamentablemente, la información pública estadística existente no permite distinguir la cantidad de sentencias a penas efectivas o a penas sustitutivas dictadas por los TOP o los JG. En efecto, solo se cuenta con información agregada respecto del tipo de pena impuesta entregada por el Ministerio Público, conforme con ello, durante 2018 se impusieron 286.878 penas a infractores de ley, de estas, $48 \%$ corresponden a multas, $35 \%$ a penas privativas y $17 \%$ a penas sustitutivas. Respecto del tipo de pena sustitutiva, conforme información estadística elaborada por Gendarmería de Chile, al 31.8.19 la institución controló a 61.274 condenados cumpliendo penas sustitutivas, de los que el 53\% estaría cumpliendo una remisión condicional, 27\% libertad vigilada en cualquiera de sus modalidades, $12 \%$ reclusión parcial, mientras que la PSBC solo estaría absorbiendo el $8 \%$ de dicha población y las expulsiones no llegarían a $1 \%{ }^{39}$.

Por otra parte, la evidencia empírica en Chile pertinente al desarrollo de la labor de juzgamiento es muy escasa. De hecho, en la actualidad solo hay unos pocos estudios y todos cuantitativos, cuatro de estos se han centrado en dar cuenta de los principales resultados de la labor de juzgamiento a partir del estudio de jurisprudencia; mientras que un quinto, más en línea con la presente investigación, se centró en analizar la labor de juzgamiento de jóvenes infractores y determinar el peso en sus decisiones de los factores legales y extralegales.

En el caso de los primeros, es decir los estudios empíricos de penas, aun cuando técnicamente no son comparables entre sí, tanto el de Hurtado y Jünemann ${ }^{40}$, el de Valdivia $^{41}$, el de Sustentank ${ }^{42}$, como el recientemente publicado por Wilenmann et al. ${ }^{43}$ encuentran similares hallazgos, esto es que la mayoría de los jueces imponían la pena mínima permitida por el marco penal -excluyendo los casos graves-, y que en los casos en que procedía sustitución de la pena privativa de la libertad, los tribunales efectivamente la decretaban.

El último estudio lo encontramos a propósito de la labor de juzgamiento de los jueces con ocasión de la Ley 20.084, desarrollado por Droppelman et al., en el que se aplicó una encuesta factorial a una muestra de JG y JO. De acuerdo con los hallazgos, la orientación hacia los fines de reinserción de la pena sí resulta significativa en el proceso de toma de decisiones de juzgamiento; mientras que la cultura judicial no parece influir en sus decisiones ${ }^{44}$.

${ }^{38}$ Estimación realizada a base de datos de sentencias del poder judicial a diciembre de 2011 extraída de www.pjud.cl, no encontrándose disponible información más actualizada.

${ }^{39}$ De acuerdo con los datos disponibles al 31.8.19 en www.gendarmeria.cl

${ }^{40}$ Hurtado y Jünemann, 2001, p. 16.

41 VAldivia, 2006, p. 38.

42 Sustentank, 2016, p. 12.

43 WilenManN et al. 2019, p. 471.

${ }^{44}$ Droppelman et al., 2017, p. 19 y 30. 
Como se observa, la literatura nacional en materia de percepciones judiciales acerca de los factores extralegales es aún incipiente, no habiéndose investigado hasta la fecha acerca de la influencia de las concepciones penológicas a la hora de sentenciar a condenados a una pena sustitutiva o la cárcel, cómo se realiza empíricamente el proceso de fundamentación y cómo controlan la ejecución de las penas sustitutivas, objetivos que motivaron la investigación que a continuación se presenta.

\section{Metodología}

El objetivo general del estudio es explorar el proceso de juzgamiento y control de la ejecución de las penas sustitutivas realizados por los jueces. Sus objetivos específicos son: conocer las preferencias penológicas de los jueces y su influencia en el proceso de juzgamiento de las penas sustitutivas, comprender el proceso de decisión y motivación (o su ausencia) de las penas sustitutivas, e indagar acerca de la labor de control de la ejecución de las penas sustitutivas.

El método utilizado en esta investigación es de tipo cualitativo, en donde la técnica de recolección de la información se basó en la práctica de entrevistas semiestructuradas a JG y JO. Las pautas de entrevistas diseñadas versaron respecto de los siguientes tópicos: carrera y cultura judicial, objetivos político-criminales de la Ley 20.603, cambios legales, fundamentación, contenido de las audiencias, y fines de las penas.

Para la determinación de la muestra de dividió el país en tres macrozonas: Norte, Sur y Centro. Dentro de cada macrozona se seleccionaron los Centros de Reinserción Social que supervisaran la mayor cantidad de penados cumpliendo penas sustitutivas y se eligieron los tribunales penales que tuvieran encomendado el juzgamiento o control de la ejecución de dichos penados.

Al 2018 habían 66 JG y 129 TOP con asiento en las jurisdicciones correspondientes a la zona macro norte (CRS Antofagasta), la zona macro sur (CRS Concepción) y la zona macro central que corresponde a CRS Santiago Sur II (Puente Alto, San Bernardo, La Pintana) y CRS Santiago Centro (Santiago, Estación Central, Quinta Normal, Independencia, Recoleta, Lo Prado $)^{45}$.

La muestra fue construida incluyendo al menos un juez de cada función en cada jurisdicción y buscando la participación paritaria de hombres y mujeres. Después de que se aseguró esa representación mínima, se asignó más peso a la muestra de JG considerando -como se explicó- que la mayoría de los casos son resueltos ante ellos, y particularmente aquellos con asiento en la zona macro central, considerando que el $80 \%$ de los jueces del marco muestral se desempeñaban en dicha zona. En total se entrevistó a una muestra de 22 jueces integrada en $77 \%$ por JG y $22 \%$ por TOP, una muestra suficiente para lograr la saturación de la información de acuerdo con los hallazgos de Guest et al. ${ }^{46}$.

\footnotetext{
${ }^{45}$ Información extraída de www.pjud.cl

${ }^{46}$ Guest et al., 2006, p. 59.
} 
El terreno fue realizado entre enero y noviembre de 2018, mediante entrevista personal. Las entrevistas fueron transcritas, codificadas en Nvivo 12 y analizadas temáticamente.

La identificación de cada uno de los entrevistados quedó definida bajo estrictos criterios de confidencialidad, por tanto, no se presentan en esta investigación los nombres específicos de las personas entrevistadas (sino que estos fueron reemplazados por un número correlativo), ni información del tribunal al que pertenecían, manteniéndose solo la distinción entre aquellas practicadas a JG o a jueces de TOP, para efecto del análisis.

\section{Hallazgos}

\section{Opciones penológicas}

A continuación se explora la percepción de los jueces respecto de la justificación de la pena en términos generales y luego se busca comprender qué sentido le atribuyen a cada pena en particular.

Los hallazgos muestran que 7 de cada 10 jueces entrevistados al ser consultados en abstracto respecto de la pena, visualizan fines de tipo mixto, particularmente el fin de retribución, seguido del de reinserción social, no existiendo diferencias entre los JG y JO. Así, si bien en la narrativa de algunos predominaba un fin por sobre el otro, la mayoría reconocía la existencia de dicha dualidad. Como indica un entrevistado:

"Yo creo que la pena tiene un componente importante de retribución, pero... desde el punto de vista criminológico, si uno dice, bueno, para qué sirve el derecho penal, solamente para castigar, o para prevenir. Y yo creo que, fundamentalmente, para prevenir otro delito, no solamente para castigar..." (Entrevista 3, JO).

Por otra parte, el fin preventivo general fue aquel que recibió el menor número de menciones, reconociendo, al parecer, que ese es un fin respecto del que, al menos, a la judicatura no le corresponde pronunciarse.

Sin embargo, esa dualidad planteada en términos generales se va desgranando a medida que se exploran sus percepciones deteniéndonos por tipo de pena, partiendo por la distinción entre cárcel y penas sustitutivas. De esta forma, efectuando dichas distinciones, 7 de cada 10 entrevistados que se pronunciaron a favor de reconocer un determinado fin a la pena de cárcel, se inclinó por concederle un fin retributivo, y reconociendo fundamentos de tipo preventivo especiales tratándose de las penas sustitutivas, como se desprende del siguiente entrevistado:

"La cárcel yo creo que es retributiva plenamente. Si es que es solamente encerrarlo actualmente, (...) y creo que toda la Ley 20.603 es preventivo especial. Partamos de esa base" (Entrevista 4, JG). 
Planteamientos de este tipo son consistentes con nuestro diseño normativo. Así, considerando el fuerte talante proporcionalista y retribucionista del nuestro código ${ }^{47}$, es esperable que la judicatura discurra en un primer momento en el fin de retribución a la hora de decidir acerca de la extensión de la pena privativa de la libertad; mientras que la concurrencia de otros fines, particularmente las justificaciones acerca de la prevención especial positiva, solo puedan operar si el legislador lo permite en el ámbito de la individualización, particularmente en la decisión de la aplicación de las penas sustitutivas en aquellos casos de penas iguales o inferiores a 5 años.

Lo anterior también resulta consistente con las percepciones particulares de cada pena entregada por los jueces entrevistados. En efecto, para 7 de cada 10 entrevistados la cárcel no era concebida como una pena que pudiera satisfacer fines de rehabilitación, reconociéndole un carácter eminentemente retributivo y en menos casos uno de tipo incapacitador, como indican los siguientes entrevistados:

_"Es que yo, en la cárcel no veo ningún factor de resocialización..." (Entrevista $11, \mathrm{JG})$.

- "La cárcel... por lo menos, la realidad nacional, no es un lugar que... Solo sirve para aislar, sacar de la sociedad un tiempo a una persona, pero más que eso no nos ayuda mucho..." (Entrevista 16, JG).

En ese sentido, es oportuno preguntarse cuál es el contenido específico que estos le otorgan a la rehabilitación o la reinserción social en el ámbito de las alternativas. Los hallazgos nos muestran que la concepción acerca de la reinserción social admite al menos 3 construcciones bajo la percepción judicial: como acceso a oferta programática, como no desocialización y como no reincidencia. Lo anterior resulta interesante, en la medida que -como se expuso anteriormente-, la reforma efectivamente recoge la primera de esas construcciones, mientras que la segunda sería más bien heredera de la conceptualización primigenia de las alternativas. Sin embargo, se desprende de las percepciones de los entrevistados esta tercera visión, también en la línea interpretativa minimalista, que supone en términos simples que el juez debe conformarse con el individuo que sea capaz de respetar externamente las leyes ${ }^{48}$, esto es, que este no reincida, siendo indiferente para efectos de su cumplimiento el que acceda a oferta de intervención o el eventual empeoramiento del contagio criminógeno que buscó evitarse con el envío a la cárcel, como se desprende de la siguiente entrevista:

"el Estado no puede regular completamente la forma de ser, ni los aspectos más acabados de la persona. En mi opinión de la pena en concreto, lo que tiene que regular el Estado, en definitiva, es que esa persona no vaya a cometer, o realizar conductas que no estén adecuadas al marco normativo" (Entrevista 12, JG).

\footnotetext{
${ }^{47}$ Cury, 2005, p. 759.

${ }^{48}$ Mir PUig, 1989, p. 37.
} 
Asimismo, otro hallazgo importante es que desde la perspectiva judicial, si bien las penas sustitutivas responden en genérico al fin de reinserción social, al indagar por tipo de pena sustitutiva, los resultados pasaban a ser complejos, atribuyéndole dicho fin solo a algunas penas sustitutivas, mas no a todas.

Así, por ejemplo, 9 de cada 10 entrevistados identificaron el fin de reinserción social con la libertad vigilada, ya sea en su modalidad simple o intensiva. Así lo expresa claramente el siguiente entrevistado:

"Yo creo que la mejor pena sustitutiva es la libertad vigilada, o la libertad vigilada intensiva. Para mí... por qué, porque creo que de verdad es la única que trabaja el tema de la reinserción” (Entrevista 10, JG).

Considerando dichos hallazgos al ser consultados por los fines que atribuían a las otras penas sustitutivas, resultó bastante difícil que los entrevistados enmarcaran sus concepciones con alguna de las justificaciones penológicas antes analizadas. Así, solo 3 de cada 10 jueces identificaron la PSBC bajo justificaciones preventivas especialespositivas, aun cuando también fue asociada a justificaciones de tipo retributivas. Por su parte, en el caso de la reclusión parcial con o sin monitoreo telemático, si bien fue identificada por varios de los entrevistados como una "formalidad", igualmente recibió algunas menciones en orden a concebirla como una buena forma de lograr la reinserción social, entendida como no desocialización, en la medida que permite a los penados mantener su trabajo en caso de estar empleados, mantenerse viviendo con su familia y su entorno, entre otras bondades. En el caso de la remisión condicional, por su parte, los entrevistados también la catalogaron como una "formalidad", considerándola insuficiente como pena para lograr el fin de reinserción social.

\section{Fundamentación versus automatismo}

A continuación se explora la percepción de los jueces respecto de la fundamentación de los requisitos objetivos y subjetivos tanto en la audiencia como a la hora de motivar su decisión. Al respecto, 6 de cada 10 JG entrevistados reconoció un automatismo a la hora de decidir de la procedencia o improcedencia de la pena sustitutiva, basados en la naturaleza negocial del sistema bajo la aplicación de los procedimientos abreviados y simplificados. Esto significa que la mayoría está a lo que soliciten las partes, y reconocen que generalmente estas no levantan debate respecto de la fundamentación de los requisitos objetivos y subjetivos, pues la pena sustitutiva es parte de lo que viene negociado. Como lo expone el siguiente entrevistado:

"Sí debo reconocer, como le decía antes, debate duro... no, no hay. Siendo bien sincero. Acá en esta zona, los fiscales raramente se oponen. Por qué, porque como son procedimientos abreviados, simplificados, en general acá en garantía, llegan con todo consensuado" (Entrevista 9, JG). 
Sin embargo, en sintonía con los hallazgos, cabría agregar que de acuerdo con la percepción de los JG lo que ilustra la práctica es que el acuerdo no solo incluye el quantum de la pena a imponer, sino que también la naturaleza de la sanción (sustitutiva), lo que claramente atenta contra la idea de la individualización de las penas.

Considerando lo anterior, resulta complejo interpretar el papel que juegan los requisitos objetivos y subjetivos en la decisión de los jueces de garantía. En efecto, si se trata más bien de una solución fruto de negociación, debiera al menos el juez hacerse cargo de que se cumpla con los requisitos objetivos en términos del quantum de la pena y del tipo de delito, para aceptar su procedencia, lo que de acuerdo con las entrevistas ocurre, en efecto. Sin embargo, lo que resulta complejo de comprender es el rol que tienen en la decisión los requisitos subjetivos y la provisión de informes de las partes que ilustren respecto de dicho requisito.

Al respecto 8 de cada 10 entrevistados que le reconocen un carácter negocial a la pena sustitutiva, reconocen solo exigir los requisitos objetivos para otorgar la pena y en definitiva fundamentar la sentencia a base de los mismos; mientras que el resto, aun cuando también le reconoce tal carácter, solicita de todas maneras informes a las partes para fundamentar la procedencia de la pena sustitutiva en relación con los requisitos de peligrosidad subjetiva, especialmente tratándose de la pena de la libertad vigilada. La razón entregada por ciertos entrevistados para lo anterior radica en la exigencia de algunos fallos de las Cortes de Apelaciones, que han resuelto que en el caso de decretar la libertad vigilada, la decisión debe ir acompañada de un informe de las partes que avale la procedencia de dicha pena. Es preciso agregar eso sí, que este requerimiento solo sería en relación con esta última pena y en algunos casos respecto de la PSBC, por lo que tratándose de la remisión condicional no se estarían exigiendo dichos informes, como se desprende de la siguiente entrevista:

“A ver, yo generalmente exijo los requisitos objetivos. En la prestación de servicios en beneficio a la comunidad, solo es una referencia a si trabaja o no, si tiene familia. En la libertad vigilada sí exijo el informe... porque hay una dificultad en la Corte, que si uno no lo pide, me revocan" (Entrevista 11, JG).

Bajo esa interpretación, cabría entonces concluir que la exigencia de informes de las partes como fundamento del cumplimiento de los requisitos subjetivos, al menos en sede de los JG, estaría cumpliendo solo un rol formal, y no estaría ilustrando particularmente en cuanto al fondo. Esta interpretación además es consistente con la poca valoración entregada por los jueces a dichos informes, toda vez que estos serían acompañados exclusivamente por la defensa, que siempre acompañaría informes favorables, restándole credibilidad a estos.

Sin embargo, esta situación no se ve reflejada tan nítidamente en las entrevistas practicadas a los jueces orales, lo que se explicaría en parte por el hecho de que la negociación no sería posible en dicha sede. De esta forma, en caso de automatismo, este no sería atribuible al carácter negocial del sistema sino más bien a la práctica judicial. En particular, algunos de los entrevistados declararon basar su decisión y por ende solo 
fundamentar basados en los requisitos objetivos, mientras que otros buscaban hacerse cargo de la fundamentación de los requisitos subjetivos, requiriendo informes de las partes, al igual que en el caso anterior, especialmente tratándose de la libertad vigilada.

Por otra parte, al ser consultados los jueces respecto de lo que la literatura reconoce como "casos al borde" (bordeline cases), 7 de cada 10 se declaró partidario de las penas sustitutivas, argumentando que, frente a la posibilidad de enviarlos a la cárcel, la pena sustitutiva era siempre una mejor opción.

"Yo... otorgo las penas sustitutivas. Generalmente las otorgo. La mayoría casi absoluta, las otorgo" (Entrevista 13, JG).

Esto de cierta forma también se explicaría por la tendencia ya analizada en torno a fundamentar solo a base de los criterios objetivos. Así, si bien la ley exige la fundamentación basada en los criterios objetivos y subjetivos, si en definitiva sean cual sean los resultados de la ponderación de estos últimos por parte del juez, estos van a optar por otorgarla, entonces los criterios subjetivos se tornan totalmente irrelevantes en la decisión judicial.

Por otro lado, estas percepciones son consistentes con los hallazgos del apartado anterior, en el sentido de que si una parte de los jueces entienden la reinserción social como no desocialización, es decir, como evitación de los efectos nocivos de la cárcel, habiéndose satisfecho las exigencias mínimas de proporcionalidad y retribución exigidas por la ley, entonces siempre primarán los criterios preventivos especiales positivos y por esta razón la cárcel no será desde esa perspectiva una opción plausible para el sentenciador.

\section{Control de la ejecución de las penas}

A continuación se explora la percepción de los jueces respecto de la labor de control de la ejecución de las penas sustitutivas en relación con las herramientas antes explicadas, aun cuando de manera tangencial serán abordados otros tópicos relevantes para su cabal comprensión.

Consultados los JG respecto de la realización de las audiencias de seguimiento, tratándose de las audiencias obligatorias a propósito de la libertad vigilada simple e intensiva, 5 de cada $10 \mathrm{JG}$ entrevistados declaró que en su tribunal se realizan audiencias de seguimiento con la periodicidad indicada por la ley, mientras que el resto declaró no realizarlas. En tanto, tratándose de las audiencias de seguimiento respecto de la pena de PSBC, ningún juez de garantía declaró realizarlas.

Entre las razones esgrimidas para su no realización se encuentra la sobrecarga de trabajo y en menor medida una crítica a la realización de audiencias en aquellos casos que han dado un correcto cumplimiento. Por su parte, aquellos entrevistados que sí declararon efectuar este tipo de audiencias, en general valoraron la instancia, como una que les permitía acercarse más humanamente al conflicto y al penado, aun cuando reconocían la existencia de diversas facetas que podía adoptar el juez en la misma. Así, más de la mitad se inclinó por reconocer un rol de autoridad o advertencia en las 
audiencias, y en menor medida un grupo reconoció asumir roles que podrían concebirse como más proactivos o de reforzamiento, más en la línea del rol asumido por los jueces en la audiencia de seguimiento de los TTD, aun cuando confesaron importantes diferencias entre dichas audiencias. Finalmente, solo una minoría de los entrevistados reconoció un rol pasivo al juez de la audiencia de seguimiento. Lo anterior se refleja en el siguiente extracto:

“...la relación del sujeto con la autoridad se refuerza con una audiencia de supervisión judicial. Porque, en definitiva, la persona que está cumpliendo una pena sustitutiva, igual tiene que responderle al sistema (...), que se institucionaliza en el tribunal, que está preocupado de que él cumpla la pena sustitutiva. Eso (..) refuerza, yo creo, la figura de autoridad, y también, la necesidad de la persona de cumplir..." (Entrevista 10, JG).

Por consiguiente, respecto de las causales de incumplimiento y revocación consagradas en la ley, 6 de cada 10 entrevistados, consistente con la visión mayoritaria de la cárcel como "último recurso" declaró frente a los incumplimientos utilizar las herramientas contempladas en la ley respecto de la intensificación de la pena sustitutiva y la posibilidad de imponer la pena siguiente más gravosa. En esa línea, los entrevistados reconocieron manejar ciertos márgenes de discreción frente a dichos incumplimientos, por ejemplo, solicitando el reingreso del condenado al CRS en caso de incumplimientos leves, la intensificación de la pena en casos de mediana gravedad o reiteración, y su revocación solo en caso de incumplimientos graves; aun cuando se reconoce la existencia de una variedad de prácticas en la materia.

Por su parte, tratándose de la posibilidad de sustituir la pena por una menos gravosa, solo un juez declaró haberla decretado, alegando una falta de proactividad de la defensa en orden a solicitarlo y del mismo tribunal en orden a actuar de oficio en la materia.

Lo anterior viene de la mano con una crítica efectuada por los jueces en torno a su falta de especialización en materia de ejecución de las penas sustitutivas como privativas de la libertad. En efecto, de las entrevistas practicadas -ya sea a JG u JO-, se pudo desprender una crítica mayoritaria a su falta de especialización para efectos de resolver materias de ejecución, a lo que se suman los argumentos relativos a la carga laboral.

En esa línea, de manera espontánea, 6 de cada 10 jueces planteó como solución a tal déficit -no una mayor capacitación o el fortalecimiento de las unidades administrativas de control de la ejecución existentes en algunos tribunales-, si no que abogaron por la creación de tribunales encargados especialmente de la ejecución o "jueces de ejecución de penas". Lo anterior se refleja en los relatos de los siguientes entrevistados:

“...establecería jueces de ejecución. Derechamente jueces de ejecución. Porque es difícil cambiar las formas de resolver. Una función adjudicativa, a casi después tutelar, y de garante de que se esté cumpliendo la ejecución. Entonces, es muy complejo a veces, hacer el cambio..." (Entrevista 14, JG). 
Lo anterior resulta importante, en la medida que existe una significativa autocrítica de los jueces penales a la labor de control de la ejecución que ellos desarrollan, dando cuenta de un desconocimiento tanto teórico como práctico de la función de hacer ejecutar lo juzgado. A esto se suma la complejidad que supone que un juez asuma dos roles muy distintos, por un lado, el del juez cuya labor principal es controlar la investigación bajo una lógica adversarial, y por el otro, el del juez que controla la ejecución.

Lo anterior se ve agudizado por el reconocimiento que efectuaron los jueces, especialmente los orales, de un desconocimiento empírico acerca del funcionamiento de las penas sustitutivas. Así, si bien estos últimos tienen encomendada por ley la labor de juzgamiento tanto de penas privativas como sustitutivas, los entrevistados fueron sinceros en reconocer su desinformación acerca de cómo funcionan en la práctica:

"En lo concreto, yo no manejo cómo se da en la práctica. Cuando nosotros en los CRS nos rinden, nos aportan informes de libertad vigilada, qué pretenden aplicar respecto de los imputados, en el papel se ve fantástico. (...) pero en la práctica no sé, desconozco cómo se desarrolla porque nosotros esa etapa no la vemos..." (Entrevista 4, JO)

Sin embargo, dicho desconocimiento se ve más matizado en el caso de los JG, en la medida que por medio de las audiencias de seguimiento - en aquellos tribunales en que son realizadas- permiten que el juez conozca un poco más de la labor ejecutada por Gendarmería de Chile, especialmente aquella realizada por los delegados de libertad vigilada. En esa línea, resulta necesario relevar la positiva opinión positiva que reflejaron los entrevistados en torno a la participación de los delegados en las audiencias, destacando la importancia de que en ella participen los profesionales encargados del caso en revisión y que no deleguen la participación en otros profesionales que no han estado en contacto con el penado. Como reconoce un juez:

"Yo me apoyo en lo que me dice el delegado. Esa es mi fuente de información confiable, y yo voy a resolver en base a lo que me diga el delegado" (Entrevista 12, JG)

Respecto de la participación en audiencias de ejecución de intervinientes como los defensores penales públicos, se encontraron opiniones diversas. Así, si bien los JG dieron cuenta transversalmente de la participación de dichos intervinientes en las audiencias de ejecución, hubo discrepancias en torno al grado de preparación de los defensores tanto en términos de la documentación acompañada como de los conocimientos técnicos para un debate más profundo en materia de ejecución, diferencias que también iban de la mano con la especialización de los defensores en la ley. Asimismo, en relación con la participación del Ministerio Público en estas audiencias, no se observó consensos en torno a la relevancia o pertinencia de la participación de este interviniente en sede de ejecución penal. 


\section{Discusión}

Los resultados dan cuenta de una posición mixta de la judicatura en lo que a fines de la pena se refiere. Sin embargo, esta dualidad se presenta no bajo una posición ius filosófica del juzgador, sino bajo un criterio de realidad en el análisis material de las respuestas jurídicas al delito. Así, se comprende la cárcel como mera retribución por carecer de componentes rehabilitadores y, en cambio, se observan las sanciones alternativas con un cariz inminentemente resocializador, en especial la libertad vigilada.

Los hallazgos no son consistentes con la literatura comparada, que en general ha dado cuenta de una inclinación de los jueces hacia los fines preventivo-especiales positivos, negando justificaciones retributivas al proceso de juzgamiento ${ }^{49}$. Sin embargo, dicha distancia puede ser explicada a base de una aparente adscripción de nuestra judicatura al modelo penológico prescrito por el CP, el que frente a las diversas teorías morales de justificación del castigo pareciera ser que se adopta una posición mixta retribucionistarehabilitadora. Este sería retribucionista, pues como se argumentó previamente, el castigo está establecido en una escala en función de la gravedad de la ofensa, reservándose la cárcel justamente para aquellas que están en la escala superior -más graves- pudiendo solo aquellas de baja y mediana gravedad ser acreedoras del sistema de sanciones alternativos a la prisión. Prueba de ello es que prácticamente la totalidad de las alternativas están previstas, en abstracto, solo para los simples delitos. A su vez, también sería rehabilitador, en la medida que, satisfecho el marco de retribución, pueda entrar a jugar un fin preventivo especial positivo plasmado, según la visión de los jueces, en las penas sustitutivas.

Es adecuado precisar, sin embargo, que el concepto de rehabilitación o de "reinserción social” adquirió diversos significados por los entrevistados, en consonancia con lo planteado por Muñoz Conde quien argumenta que "el término resocialización se ha convertido en un modewort, en una palabra de moda que por todo el mundo se emplea y no solo entre los juristas, sin que nadie sepa muy bien lo que quiere decir con ello" 50 .

Lo interesante en las diversas concepciones que tienen los jueces acerca de la reinserción social, es que en el caso de aquellos que la conciben como "el acceso a una oferta programática”, esta se traduce necesariamente en acciones positivas por parte del Estado (educación, capacitación laboral, programas psicosociales, etc.). En cambio, las dos acepciones restantes (no desocialización y no reincidencia) se traducen más bien en una obligación de no hacer, que de cierta forma reconocen el carácter autonómico de la persona, la que cumple con los fines de prevención, en la medida que no se aparte de su medio o que sencillamente no cometa nuevos delitos.

Reconociendo dichas percepciones respecto de los fines de la pena, resulta necesario saber cómo en el quehacer sentenciador pesan dichos fines. Al respecto, los hallazgos dan cuenta que dichas percepciones parecieran no jugar un papel importante en la decisión judicial de otorgar una pena sustitutiva, nuevamente en disonancia con la literatura

\footnotetext{
${ }^{49}$ Hogarth, 1971, p. 18; Mackenzie, 2005, 83 y ss.

${ }^{50}$ Muñoz Conde, 1979, p. 75.
} 
empírica revisada ${ }^{51}$. Esto parece explicarse por la tendencia a la automatización declarada por los jueces, en la que estos parecen más bien ser recipientes de un proceso de negociación, impidiendo la realización del proceso de individualización de la pena. Dichos resultados son a su vez consistentes con lo planteado por Riego, quien argumenta que si bien el modelo de enjuiciamiento chileno es adversarial, también presenta fuertes rasgos de justicia negociada, mediante la posibilidad del plea barganing contemplada a propósito del procedimiento abreviado, caso en donde el juez queda sujeto en cuanto al máximo de la pena a imponer a la acusación del fiscal, la que a su vez es furto de la negociación previa de las partes como contracara a la admisión de responsabilidad por parte del imputado 52 .

A lo anterior, además, se suma una preconcepción pragmática revelada por la magistratura de que la cárcel debe ser el "último recurso" debido a las condiciones existentes en ellas, hallazgos que serían similares a aquellos encontrados en la literatura revisada, especialmente lo planteado por Tata, en el sentido de entender el juzgamiento como parte de una secuencia en el proceso de decisión, en donde el juez es un miembro colaborativo del "mundo del juzgamiento" (sentencing world) ${ }^{53}$, siendo un "actor social" más, permeable por cierto a las lógicas de negociación que han ido ganando terreno progresivamente en el proceso penal chileno; y también al discurso de la cárcel como "último recurso" 54 .

Sin perjuicio de lo anterior, pareciera, eso sí, que estas finalidades de las penas y del castigo adquieren mayor peso relativo a la hora de decidir aquellos casos borderline, o sea, aquellos en que se discute la ejecución de una pena de prisión o su sustitución por una alternativa, el que si bien no deja ser automático, supone al menos el ejercicio de la adopción de una adscripción penológica más clara en favor de la rehabilitación.

Sin embargo, desde la perspectiva de la fundamentación, en sintonía con los anteriores hallazgos, también fue posible encontrar un automatismo en cuanto al cumplimiento formal tanto de los requisitos objetivos como de los requisitos subjetivos, traducida en una inexistente o insuficiente fundamentación de la resolución judicial que se pronuncia por la procedencia de la pena sustitutiva. Es acertado resaltar que dichos resultados estarían en línea con lo encontrado por Wilenmann et al., quienes dan cuenta de una elevada estandarización de las decisiones de otorgamiento de los jueces orales en aquellos casos en que resulta procedente una pena sustitutiva, fundada esencialmente en la concurrencia de los requisitos objetivos 55 .

En ese sentido, si bien en nuestro sistema la motivación de las sentencias, en todos sus aspectos, es un deber para el juzgador ${ }^{56}$; el proceso de sentenciamiento -entendido

${ }^{51}$ Hogarth, 1971, p. 68 y ss; Mackenzie, 2005, p. 89 y ss; De Castro Rodríguez et al., 2019, pp. 174-175.

52 Riego, 2017, p. 1086 y ss.

53 TATA, 2007, p. 442.

54 TATA, 2016, p. 24.

55 WilenMANN et al. 2019, p. 474.

56 Véase artículo $19 \mathrm{~N}^{\circ} 3$ inciso $5^{\circ}$ de la Constitución Política de la Republica; artículos $1^{\circ}, 4^{\circ}, 5^{\circ}$, $297^{\circ}$ y $342^{\circ}$ letra c) y d) del CPP. 
como un proceso colaborativo, y en nuestro sistema, con un marcado acento en carácter negocial, sumado al reconocimiento generalizado de la cárcel como un lugar de "último recurso"-, torna compleja la labor de fundamentación cuando la solución sistémica viene más bien por default, que por un proceso axiológico de fundamentación, en sintonía con lo planteado por Tata $^{57}$. Tampoco ayuda la interpretación de los requisitos subjetivos previstos por el legislador a propósito de las alternativas, los que, lejos de facilitar la labor de juzgamiento en una decisión dicotómica -la prisión o una pena sustitutiva-, exige aptitudes pitonisas, de poder identificar, sin los insumos adecuados, si el condenado delinquirá o no en el futuro.

Por su parte, la valoración positiva del control de la ejecución de las penas debe ser interpretada bajo el reconocimiento histórico, de un rol más bien limitado en la supervisión de las penas, excepto cuando el penado no cumplía con sus obligaciones y debía retornar por ello al juzgado para proceder por su incumplimiento. Sin embargo, a propósito de la reforma legal de las penas sustitutivas, particularmente a la finalidad de mayor control que la tiñe $e^{58}$, esta situación ha empezado a cambiar, configurándose la supervisión como una de sus innovaciones principales.

Estos hallazgos se encuentran en sintonía con diversos estudios criminológicos que han subrayado la relevancia del papel del juez en el proceso de desistimiento de los infractores, relacionada con la asunción de un rol más activo y próximo al penado en el proceso de supervisión ${ }^{59}$. Así, los jueces al tener una participación más activa, les permite poder identificar y abordar mejor los problemas que subyacen al comportamiento delictivo de los infractores ${ }^{60}$. Además, hay evidencia de que la interacción con los jueces contribuye a aumentar las percepciones de justicia de los penados ${ }^{61}$, la que redunda en una mayor legitimidad de los actores legales y una mayor disposición por parte de los infractores a cumplir con las instrucciones que reciben y a vivir conforme a derecho ${ }^{62}$.

En ese sentido, la presente investigación da cuenta del llamado efectuado por los propios jueces, de regular de mejor forma la fase de ejecución, de dotarla de jueces exclusivamente abocados a su conocimiento, que supere el rol binario y de cierta forma contrapuesto, de juzgador (bajo un rol adversarial) y de controlador de la ejecución (bajo un rol de apercibimiento y/de refuerzo).

\section{Conclusiones}

La presente investigación constituye un esfuerzo por entregar conocimiento empírico acerca de la labor de juzgamiento y de control de la ejecución ejercida por los

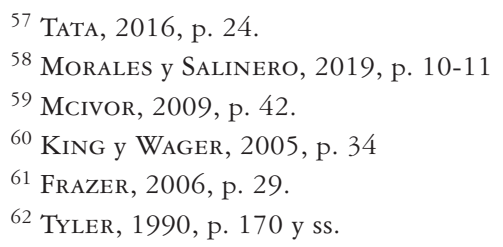


jueces, a propósito de las penas sustitutivas; el que, si bien en lo jurídico pareciera ser un proceso sin mayor dificultad, en el análisis de sus supuestos de procedencia presenta complejidades que no pueden ser comprendidas solo bajo un análisis normativo.

Considerando dicho contexto, si bien la investigación buscó comprender al alero de las concepciones penológicas el proceso de juzgamiento de las penas sustitutivas -en sintonía con lo reportado por las investigaciones comparadas-, los hallazgos dieron cuenta de un proceso mucho más complejo en el que dichas posiciones tendrían un rol de menor relevancia. Este proceso caracterizado por la influencia de un modelo de corte negocial sumado a una preconcepción pragmática de que la cárcel debe ser el "último recurso" debido a las condiciones existentes en ellas, ha impactado fuertemente tanto el rol de decisión acerca de la procedencia de las penas sustitutivas como su fundamentación, tornándola en uno dotado de automatismo y fundamentación formal de los requisitos de procedencia, en una trama más compleja de interacción entre los actores.

En ese sentido, a la luz de la discusión de un nuevo CP, resultará necesario considerar estos hallazgos y buscar la forma de repotenciar la labor de juzgamiento y particularmente de individualización de las penas, en sintonía con una revalorización de la fundamentación a la hora de determinar las penas a aplicar. Lo anterior se logrará en la medida que se pueda establecer un catálogo de penas principales tanto privativas como alternativas, dentro de estas, una vez satisfechos criterios mínimos de retribución ${ }^{63}$, se pueda desplegar la labor individualizadora del juez, que permitan atender a las necesidades de intervención del infractor, mediante la elección de entre un abanico de sanciones que permita al juez elegir la pena idónea.

Asimismo, los hallazgos en materia de ejecución penal, dan cuenta de la necesidad de avanzar decididamente en la especialización en esta materia, con un cuerpo normativo y actores que estén capacitados en las consideraciones criminológicas, y que revalorice esta función a partir de la propia experiencia que hoy nos entrega el control de ejecución de la Ley 20.063.

\section{BIBLIOGRAFÍA}

Ashworth, Andrew, 2010: Sentencing and Criminal Justice, $5^{\circ}$ ed., Cambridge, Cambridge University Press.

Biblioteca del Congreso Nacional, 2012: Historia de la ley $N^{\circ}$ 20.603. Valparaíso, Biblioteca del Congreso Nacional.

Cury, Enrique, 2005: Derecho Penal. Parte General. $8^{a}$ ed, Santiago, Ediciones Universidad Católica de Chile.

De Castro Rodrigues, Andreia, Sacau, Ana, de Oliveira, Jorge. Q., Gonçalves, Rui, 2019: "Prison sentences: last resort or the default sanction?", Psychology, Crime E Law, 25(2), 171-194.

\footnotetext{
${ }^{63}$ Von Hirsch, 1998, p. 100.
} 
Droppelman, Catalina, Carvacho, Pablo, Mateo, Muriel, Valenzuela, Eduardo, 2017: Estudio sobre los factores que influyen en las decisiones judiciales de la justicia juvenil en Chile, propuesta desarrollada en el marco del Concurso de Políticas Públicas UC, Santiago.

Feijoo, Bernardo, 2007: Retribución y Prevención General. Un estudio sobre la teoría de la pena y las funciones del Derecho Penal, Buenos Aires, B de F.

Frase, Richard, 2005: "Punishment Purposes. A More Perfect System: Twenty-Five Years of Guidelines Sentencing Reform”, Stanford Law Review, 58 (1), 67-83.

Frazer, Somjen, 2006: The impact of the community court model on defendant perceptions of fairness: A case study of the red hook community justice center, Nueva york, Centre for Court innovation.

Garland, David, 1990: Castigo y sociedad moderna: un estudio de teoría social, Madrid, Siglo xxi.

Guest, Greg, Bunce, Arwen, Johnson, Laura, 2006: "How many interviews are enough? An experiment with data saturation and variability", Field methods, 18 (1), 59-82.

Halliday, Simon, Burns, Nicolas, Hutton, Neil, Mcneill, Fergus, Tata, Cyrus, 2008: "Shadow writing and participant observation: a study of criminal justice social work around sentencing”, Journal of Law and Society, 35(2),189-213.

Honvitz, María Inés, López, Julián, 2003: Derecho procesal penal chileno, Tomo I, Santiago, Editorial Jurídica de Chile

Hogarth, John, 1971: Sentencing as a Human Process, Toronto, University of Toronto Press.

Hough, Mike, Jacobson, Jessica, Millie, Andrew, 2003: The Decision to Imprison: Sentencing and the Prison Population, London, Prison Reform Trust.

Hutton, Neil, 2006: "Sentencing as a Social Practice”, en Armstrong, Sarah, McAra, Lesley (Eds), Perspectives on Punishment, Oxford, Oxford, University Press.

Hurtado, Paula, Jünemann, Francisca, 2001: Estudio empírico de penas en Chile, Santiago, Fundación Paz Ciudadana.

KING, Michael, Wager, Julie, 2005: "Therapeutic jurisprudence and problem -solving case management", Journal of Judicial Administration, 15, 28-36.

Lippke, Richard, 2006: "Mixed Theories of Punishment and Mixed Offenders: Some Unresolved Tensions", The Southern Journal of Philosophy, 44(2), 273-295.

Lovegrove, Austin, 1999: "Statistical Information Systems as a Means to Consistency and Rationality in Sentencing", International Journal of Law and Information Technology, 7, 31-72.

Mcivor, Gill, 2009: "Therapeutic jurisprudence and procedural justice in Scottish”, Criminology and criminal justice, 9 (1), 29-49.

Mackenzie, Geraldine, 2005: How judges sentence, New South Wales, Federation Press.

Maguire, Niam, 2014: "When is prison a last resort? Definitional problems and judicial interpretations", Irish Criminal Law Journal, 24(3), 62-72.

Medina, Juan José, 2011: Políticas y estrategias de prevención del delito y seguridad ciudadana, Madrid, Edisofer,

Mir Puig, Santiago, 1989: ¿Qué queda en pie de la resocialización?, Cuaderno del Instituto Vasco de Criminología, San Sebastián, 2.

Morales, Ana María, Salinero, Sebastián: "Fundamento político criminal y naturaleza jurídica de las penas alternativas en Chile", Revista Chilena de Derecho (aceptado, en prensa).

MuÑoz Conde, Francisco, 2019: "La resocialización del delincuente: Análisis y critica de un mito", Cuadernos de Política Criminal, No 7, 73-84.

Salinero, Sebastián, Morales, Ana María, 2019: "Las penas alternativas a la cárcel en Chile. Un análisis desde su evolución histórica", Revista de Derecho de la Pontificia Universidad Católica de Valparaíso, 52, 1-38.

SpoHn, Cassia, 2000: "Thirty Years of Sentencing Reform”, NIJ Criminal Justice, 3, 427-501. 
SpoHn, Cassia, 2009: How do judges decide - The search of fairness and justice in punishment, Thousand Oaks, Sage Publications.

Sustentank, 2016: Informe final Estudio empírico de penas, Santiago, Ministerio de Justicia y Derechos Humanos.

TATA, Cyrus, 2016: "How can prison sentencing be reduced in Scotland?", Scottish Justice Matters, $4(1), 23-24$.

TATA, Cyrus, 2007: "Sentencing as craftwork and the binary epistemologies of the discretionary decision process", Social \& Legal Studies 16(3), 425-447.

Tyler, Tom, 1990: Why people obey the law, New Haven, Yale University Press.

Riego, Cristián, 2017: "El procedimiento abreviado en la ley 20.931", Política criminal, 12, (24), 1085-1105.

Roxin, Claus, 2006: Derecho Penal. Parte General. T. I, $2^{\text {a }}$ Ed. Madrid, Civitas.

Ulmer, Jeffery, (2012): “New Directions in Sentencing”, Justice Quaterly,29, (1), 1-40.

VAldivia, Claudio, 2006: "Estudio empírico de penas en Chile 2006”, en ISUC y Fundación Paz Ciudadana Tercer simposio internacional sobre violencia y delincuencia.

Velásquez, Javier, 2018: Doing Justice: Sentencing Practices in Scottish Sheriff Courts (Tesis doctoral). Edimburgh, University of Edimburgh.

Von Hirsch, Andrew, 1983: Censure and Sanctions. Oxford, Clarendon Press.

Wilenmann, Javier, Medina, Francisco, Olivares, Esteban, Del Fierro, Nicolás, 2019: "La determinación de la pena en la práctica judicial chilena”, Política Criminal, 14 (27), 456-490. 
